\title{
Cultura Innovativa: Prospectiva de las condiciones estratégicas para el desarrollo del capital humano
}

\author{
Innovative Culture: Prospects of the strategic conditions for the development of human capital
}

\section{Yan Carlos Ureña Villamizar \\ Postdoctor en Ciencias Humanas \\ Universidad del Zulia, Maracaibo, Venezuela yan_urena@hotmail.com \\ Iris Agustina Jiménez Pitre Doctora en Ciencias Mención Gerencia Universidad de la Guajira, Colombia iajimenez@uniguajira.edu.co \\ Aquiles Mejía Anaya Magíster Scientiarum en Gerencia de Proyectos de Investigación y Desarrollo Universidad Rafael Belloso Chacin, Venezuela amejaya@gmail.com \\ Jasón Fred Vilarete Molina Magíster Scientiarum en Gerencia de Proyectos de Investigación y Desarrollo \\ Diebold Colombia S.A., Colombia villarette70@hotmail.com}

Tipología: Artículo de Reflexión Fecha de Recibido: Julio 09 de 2014

Fecha de Aceptación: Septiembre 02 de 2014

Para citar este artículo: Ureña, V. Y., Jiménez, P. I., Mejía, A, A., \& Vilarete, M, J. (2014). Cultura innovativa: prospectiva de las condiciones estratégicas para el desarrollo del capital humano, Clío América, 8 (16), 195 - 200
Resumen: En toda entidad el desarrollo de la cultura ayuda al crecimiento de lo novedoso, permitiendo que las actividades se lleven de forma fácil y acertadas dejando generar conocimientos manejados por cada uno de los individuos. Tomando en cuenta la realidad de las empresas la cultura innovativa ayuda a mejorar así como generar alternativas en la tecnología utilizadas en las cuales se adaptan los valores y conocimientos aunque muchas veces los empleados se rehúsan a los cambios generando conflictos internos y gerenciales, ahora bien, a nivel latinoamericano se limitan en cumplir con lo establecido en la misma sin tomar en cuenta los avances y cambios tecnológicos que ayudarían al desarrollo de las actividades comerciales y gerencial. En consecuencia, en la industria Bancaria la cultura innovativa se encuentra relacionada con el desarrollo productivo de la misma en donde se toma en cuenta los conocimientos de cada individuo así como de los cambios generados en la tecnología para mejorar las actividades comerciales. Este aspecto considera los planes de mantenimientos preventivos, correctivos, entre otros, en la línea completa de servicios integrados, soluciones de seguridad, y administración de red, que al parecer, sus funciones se ven afectadas por la necesidad de logro, creatividad, pensamiento estratégico.

Palabras clave: Cultura, Innovación, Capital, Recuso Humano.

JEL: 031, 032.

Abstract: In any entity the development of culture helps the growth of the new, allowing activities to be carried easily and successful leaving generate knowledge managed by each one of the individuals. Taking into account the reality of the company's innovative culture helps to improve as well as build on technology used in which adapt values and alternatives expertise although many times employees refuse to changes generating internal and management, conflicts now, Latin America are limited to comply with it without taking into account the advances and changes in technology that would help the development of the commercial and managerial activities. Consequently, in the banking industry innovative culture is related to the development of production of the same where you take into account the knowledge of each individual as well as the changes generated in technology to improve business. This aspect is considered plans of preventive maintenance, corrective, among others, the full line of integrated services, and security solutions, network management, apparently, its functions are affected by the need for achievement, creativity and strategic thinking.

Keywords: Culture, Innovation, Capital, Human Resources. 


\section{Introducción}

En la actualidad existen cambios, evoluciones en lo que corresponde a la teoría de sistema aplicada a las empresas, tomando en cuenta como enfoque principal los postulados de Senge, (1990). Donde señala la aplicabilidad de diferentes ámbitos que envuelven a la organización pilar fundamental del crecimiento humano. Asimismo, los postulados del autor antes mencionado permiten a la empresa identificarse bajo conceptos escritos como mentales, los cuales se utilizan normalmente en la organización tales como las normativas y políticas internas de la misma. Por otra parte, es necesario tomar en cuenta que en toda organización las personas deben considerar que su labor es lo más importante permitiendo de esta forma el crecimiento de cada uno de ellos $y$, por ende, del sistema.

Ahora bien, una organización inteligente se encuentra basada en la teoría desarrollada por Senge, (1990). La Quinta Disciplina, donde se denota que esta es cada vez más consciente de su base de conocimientos, sobre todo el acopio de conocimiento tácito en el corazón y la mente de los empleados. De la misma forma, en la organización inteligente la gente crece fervientemente en el pro de la misma demostrando que cada uno de los que labora allí se encuentran inmersos dentro de proyectos de aprendizajes constantes, claramente, para que exista una organización inteligente cada individuo actualiza sus conocimientos integrando el trabajo en equipo además de utilizar el dominio personal y adecuando todos el mismo modelo mental que permita mejorar el ecosistema empresarial.

En este aspecto, para alcanzar el capital intelectual en una institución se debe perfeccionar al capital humano, desarrollando así los activos intangibles con que cuentan los participantes (principios, cualidades, destrezas y sabiduría), consecutivamente organizar ese capital, en otros términos, persuadir al cuerpo laboral a colaborar, gestionar y documentar de forma segura el talento que ha logrado, de la misma manera aparecer el aspecto relacional como sustento positivo de la socialización. En este momento, el contexto competitivo anhela transformaciones, la rapidez con que aparecen, compiten $\mathrm{y}$ fallece una idea es lo que ha dado surgimiento a un estilo de gerencia en las entidades de modo muy diferente a como se realizaba inicialmente.

Es decir, el capital intelectual se puede agrupar de acuerdo con los ámbitos de trabajo de la formación en otra matriz diferente de la habitualmente encontrada en los manuales clásicos sobre la organización en la empresa, o dicho de otra manera, puede ofrecer la posibilidad de añadir otras matrices necesidadesacciones-formativas a las que se está acostumbrado a trabajar, el Modelo Sistémico Organizacional representan las condiciones que darán forma a las relaciones de un mundo lleno de información y conocimientos donde las posibles externalidades afectan directa e indirectamente la labores empresariales, la calidad de los esquemas de trabajo personal así como de organización tienen que ver con la adaptabilidad a las situaciones de riesgo e incertidumbre de la empresa en cuanto a la optimización de sus procesos, lo cual conlleva a mejorar el ritmo de la entidad y una calidad laboral.

En síntesis para el investigador, el Modelo Sistémico Organizacional, es un componente de gran relevancia en una organización, lo cual representa el valor que se requiere para el trabajo colaborativo. Asimismo, es un nuevo factor de competitividad en el área del conocimiento y que requiere ir más allá del entorno en que se desenvuelve incluyendo lo dinámico, flexible y elasticidad a los procesos organizacionales, además de apoyar sus bases en el foque tecnológico (capital digital), para poder enfocarse en la capacidad creativa sistémica para promover la innovación en las diferentes áreas de la ecología organizacional, apoyada en la construcción de nuevos enfoque empresariales.

\section{Cultura Innovativa}

La cultura, comprendida de reglas no escritas, creencias compartidas y modelos mentales de las personas, afecta la eficiencia de los instrumentos de innovación que se pueden describir con el desarrollo de las actividades de la empresa. Ahora bien, la cultura de la empresa valoraba mucho la homogeneidad y el cumplimiento de los requisitos, y la compañía no podía trabajar con la velocidad de cambio a su alrededor. La cultura no es estática, evoluciona constantemente. Para desarrollar la 
cultura, la empresa debe diseñar nuevos sistemas, procesos, nuevos símbolos y valores corporativos.

En el mismo sentido, Galván \& García, (2007) afirman: "Establecer un marco económico y un clima social y cultural adecuado a la concertación con los agentes sociales, al surgimiento de personas emprendedoras y propicio al desarrollo de actividades innovadoras" (p.106). Así, en muchísimas oportunidades podemos evidenciar cómo es la versatilidad del personal en el instante en que se cruza al interior de las organizaciones. Desde esta óptica general, merecería expresar que las compañías ligadas con el éxito se hallan disponibles hacia la innovación y el conocimiento. Lo cual denota crear las facilidades para fomentar en el personal un aprendizaje continuo y estimarlas como el activo primordial de la sociedad.

En esencia, la cultura innovativa influye en la motivación, en el desempeño y la satisfacción con el trabajo y la actualización tecnológica de una empresa. Los empleados esperan ciertas recompensas, estímulos y hasta frustraciones, basados en la percepción que tienen de la cultura innovativa de la empresa. La cultura deriva en un sistema de comportamiento empresarial que incluye la filosofía así como las metas y el liderazgo, aproximándose estos elementos en un sistema de controles que interactúan con las actitudes personales, factores motivacionales y desarrollo tecnológicos a los empleados.

\section{Condiciones para la Innovación}

La gestión y la cultura innovadora entraña ideas radicales. Algunos aspectos estratégicos son clave y necesarios para implementarla con éxito. Ahora bien, la innovación está ligada a todas las condiciones de competitividad; en este punto, se convierte en un proceso fundamental para alcanzar la idoneidad en el mercado global Entre tanto, se alcanza un nivel competitivo sin la necesidad de tener innovación, conservando sin lugar a dudas un proceso de mejora continua, pero estos no logran ser idóneos cuando el mercado se halla inundado, cuando existe una alta demanda y cuando florecen requerimiento que los servicios ò productos actuales no consiguen resolver. En consecuencia, Hidalgo, León \& Pavón, (2004) expresa que las condiciones para la innovación son las circunstanciasen las que una empresa maneja la entrada del descubrimiento ya sea tecnológica como de conocimientos, donde se toma en cuenta al capital que labora y se encarga del manejo de la información es decir sus cualidades características.

En otras palabras, las condiciones para la innovación son las circunstancias donde lo novedoso se maneja de forma inteligente permitiendo que exista una interacción entre los avances tecnológicos de la empresa lo cual trae como consecuencia las labores de calidad. Por su parte, Escorsa, (2005) expresa que las condiciones para la innovación es la situación en la que se debe desenvolver las empresas que se encuentran adaptándose a los cambios de innovación. Por otra parte, las incertidumbres tecnológicas y del mercado asociadas con las innovaciones radicales, hacen a veces esta detección difícil.

Finalmente, en el contexto actual las innovaciones conlleva una gran incertidumbre, y muchas veces el comportamiento de los gerentes viene sugestionada por los presupuestos de las quiebras tecnológicas en términos económicos lo cual no lo torna difícil sino casi imposible. De igual forma, la suposición que cohabita en el entorno de la innovación se halla en el éxito de la aceptación de esta tecnología radical y es decreciente con el tiempo. El incremento sostenido en el tiempo del capital financiero requiere discontinuidades tecnológicas.

A) Creatividad: Es tal vez el comienzo de toda innovación popularmente conocida y más implementada, por esto, implica forjar nuevos objetos 0 ideas que no existían anteriormente. La creatividad encauzada a través de una disposición lógica, da como consecuencia innovaciones de éxito. La innovación, a su vez, tendrá una mayor repercusión en la posición competitiva cuanto más contribuya a diferenciar de la competencia, en donde se toma en cuenta la creatividad como una condición esencial para la innovación.

Al respecto, Rodríguez, (2004). "La creatividad se estimula en un ambiente de tolerancia que aliente la exploración de nuevas ideas y formas de hacer las cosas" (p.58). Establece el punto focal de diferentes tipos de esquemas para administrar la innovación, la 
creatividad es estimada desde mucho tiempo atrás como pilar fundamental y requerido para innovar, pese a que, cuenta con características esenciales. Por ello, no podemos decir que a una idea creativa es pie para generar una invención; los pensamientos altamente significativos instauran las bases fundamentales para la innovación. El miedo y la debilidad espantan la creatividad o por lo menos la hacen menos probable, el hecho de que el gerente se llene de valor y deshacerse del temor al rechazo, a la autoridad, a la burla, al ridículo, facilita el dejarse atraer por lo novedoso, lo inhabitual, lo misterioso, lo ambiguo, lo contradictorio y lo inesperado.

En efecto, se puede inferir que la creatividad como condición para la innovación permite al sujeto solucionar situaciones que se generan en la acción innovativa. En otras palabras, la creatividad es el proceso imaginativo, audaz e innovador que tiene el gerente que va a desarrollar la acción, permitiendo tener amplias ideas pero originales. Asimismo, esta condición está dispuesta a romper moldes, y a aprender hasta lograr los propósitos esperados.

B) Pensamiento Estratégico: Se entrelazan particularidades diferentes y complejas pero, posiblemente, uno de los atributos más significativos es que puede ser asimilado, es decir, que destinando en conocimiento se pueden convertir el llamado pensamiento tradicionalista en pensamiento estratégico. Un correcto pensamiento estratégico debe iniciar invariablemente de la misión de la compañía la que a su vez se enfoca a una perspectiva de futuro integrando valores, asentado en lo versátil de la realidad, y sobre todo materializando la cultura organizacional tácticamente.

Por su parte, Monteagudo, (2005) expresa que el pensamiento estratégico de una empresa es la relación de innovaciones, audacia, habilidades que se manejan bajo una representación tradicional, el cual le permita a las organizaciones adelantar así como prosperar hacia el futuro de una manera agradable para todo el capital humano que labora allí. Ahora bien, esta condición para la innovación permite que llegue a acuerdos los empleados y gerente, así como entre las metodologías que desenvuelven la acción como tal.
Tomando en cuenta lo planteado, Según Morrisey (1996), “el pensamiento estratégico individual incluye la aplicación del juicio basado en la experiencia para determinar las direcciones futuras (...) es la coordinación de mentes creativas dentro de una perspectiva común que le permita a un negocio avanzar hacia el futuro de una manera satisfactoria para todos" (p. 56). Igualmente, con el manejo del pensamiento estratégico se puede tener una efectiva toma de decisiones frente a situaciones que traigan peligro a la generación de conocimientos innovadores. La intención es apoyar e indagar los diferentes retos del futuro, tanto predecible como inadvertido, más que disponerlo para mañana diferente, alcanzando las metas propuestas.

El pensamiento estratégico difiere firmemente del convencional cuyas técnicas de razonamiento está basado en la linealidad y la reproducción del pensamiento mecanizado, de afirmación visible, que designa una medida estable ante contextos similares, diferenciando con el conocimiento básico. De alguna forma es un aporte necesario y auténtico en términos estratégico del pensamiento

C) Sensibilidad al Cambio: Generalmente, los directivos empresariales estiman la calidad del nivel de medición en aplicación a su valor y su fiabilidad. No obstante, la susceptibilidad a las transformaciones debe contemplarse como una particularidad primordial de las herramientas de evaluación, creados para calcular una innovación longitudinal en el transcurso de un período. De igual forma, la sensibilidad al cambio se explica en aquella facultad con que cuenta una dimensión para variar en un transcurso de un tiempo establecido; se ocupa de la eventualidad de descubrir alguna variación de tipo estadístico (Husted, Cook, Farewell\& Gladman, 2000).

Tomando en cuenta lo anterior, la sensibilidad al cambio viene dada por la flexibilidad que tiene el gerente así como los empleados para adaptarse a los cambios que genera la innovación así como la modificación de los elementos de la tecnología para luego implementarlo en la acción empresarial. En consecuencia, la sensibilidad al cambio es la facultad para admitir con naturalidad al mismo tiempo afrontar con elasticidad y modo cambiable a 
eventos e individuos desconocidos con anterioridad, de esta forma someterse a las variaciones con una conducta objetiva y benéfica (Monteagudo, 2005). Similarmente, podemos observar la sensibilidad al cambio como el estado al que se llega en distintas derivaciones sobre la base de estudios practicados a un experimento idéntico en el momento que, se ha generado una variación actual en el aspecto corporativo.

La sensibilidad al cambio deriva de la innovación $y$ del contexto en que se emplea el mecanismo de medición. Por ende, la alternativa del modelo de análisis para la innovación y de los coeficientes de sensibilidad al cambio obedece esencialmente de las características del prototipo, principalmente la elaboración del diseño y del cambio a esperar. De la misma manera, la sensibilidad al cambio penderá así del proceso utilizado como de la dimensión de resultado aplicada para establecer la validez de la innovación. La sensibilidad al cambio externa es la escala con que estos en una proporción de tiempo se interrelacionan con cambios ajustados en un nivel estándar a la etapa de la innovación.

D) Adaptación al Riesgo: En la búsqueda de transformaciones corporativas es imprescindible tratar de revolver los riesgos que se manifiesten sin quitarle el valor que tienen y procurando esparcir la seguridad empresarial dado que esto no admite dar una perspectiva tan primordial para la consecución los objetivos planteados. Al respecto, Pozo, (2004) muestra que la adaptación del riesgo es la aceptación de las normas de innovación por la empresa. La adaptación al riesgo se logra cristalizar cuando no hay apropiados programas de gerencia, accidentalidad, fiscalización de la producción tecnológica coherente a los planes, procesos, bienes o servicios.

Efectivamente, admitir una condición tratando de lograr el mayor éxito pero aceptando riesgos o en contraste adoptando por la simplicidad de opciones que no sean más promisorias pero que alcanzan la confianza de cumplir con una finalidad. Señala Escorsa, (2005) que la adopción del riesgo permite el desarrollo del impacto y la probabilidad de ocurrencia de una situación. Entre todos los factores que permitirían caracterizar una adaptación al riesgo, dos de ellos, el impacto y la probabilidad de ocurrencia, son los que tienen mayor importancia para el gestor. La adaptación al riesgo como el ajuste que hace el sujeto investigador a las diferentes situaciones que orienten riesgo en la acción investigativas. Para el contexto de riesgo los datos que se tiene para arreglar una dificultad es insuficiente, es decir, se vislumbra el problema, se observa las posibles soluciones, pero no se advertir con seguridad las consecuencias que se obtendrán. De igual forma, cuando las compañías financian tardíamente $y$, en ciertos momentos, sin ensayar apropiadamente las tecnologías. La primordial acción que debe desplegarse en la organización cuando se están realizando los planes estratégicos es alcanzar que todas las decisiones de la gestión de riesgo estén alineadas con los objetivos empresariales.

\section{Conclusión}

Se debe perfeccionar las condiciones y las dinámicas culturales para la innovación, la cuales deben ser colocadas en marcha para la empresa Diebold Colombia S.A, el objetivo principal es dar cumplimiento a las estrategias para posibilitar la edificación de una cultura basada en la innovación, impulsando los agentes medibles a corto, mediano y largo plazo de las metas, las estrategias operativas, la afiliación cultural como componente del cambio tecnológico y de la innovación como la destreza global de la empresa Diebold Colombia S.A, favoreciendo el posicionamiento de ventajas diferenciadoras en alusión a la competencia externos e internos, impulsando la creación de segmentos del productos para acrecentar los beneficios sociales, ambientales, entre otros.

En este aspecto, las acciones favorecen a incrementar el conocimiento en lo actual e innovador. Aquí habita la clave para el adecuado manejo tecnológico de la empresa para mejorar su rendimiento económico. En atención a las consideraciones el investigador apoyado en las teóricas conjuntamente con su análisis expone:

a) Desarrollar estudios de potencialidades que identifiquen sistemáticamente y evalúen las propuestas de acción practica que se pueden emprender, considerando requerimientos efec- 
tivos y legítimos del estado real de la empresa, siguiendo líneas de acción, a través de talleres participativos.

b) Realizar un modelo de Flexigrama con concepción actualizada y participativa para la gestión y desarrollo de la empresa, basado en el aprendizaje, para la generación participativa como sistemática de innovaciones, entendida desde el desarrollo de procesos permanentes de cambio que conduzca a nuevas prácticas en el pensamiento sistémico prospectivo.

c) Promover planes de sensibilización al gremio empresarial, con la propósito de adiestrar a los trabajadores para interiorizar la cultura innovativa como prerrogativa de profesionalismo en la procesos organizacionales, promoviendo las destrezas en rutinas diariamente por medio de doctrinas, conductas, normas, métodos y técnicas de estímulos, como pautas de interrelaciones con sus empleados, reconociendo la cultura organizacional como mecanismo como reformadora de conductas del personal que logran acceder u obstaculizar la variabilidad, y el ajuste a los cambios y al talento empresarial.

d) Experimentar nuevas formas de trabajar, desarrollando nuevos productos o mejorando los ya existentes, en función de elevar el nivel de capacidad y la motivación de las persona. Queriendo decir con esto, que los dos aspectos, van entrelazados para que de esta manera se establezca un buen desempeño del trabajador, influyendo sobre el rendimiento de las personas, debido a la motivación que refiere a los estados internos que energizan y dirigen la conducta hacia las metas

\section{REFERENCIAS BIBLIOGRÁFICAS}

Escorsa, P. (2005). Inteligencia Competitiva y Transferencia de Tecnologías: Reflexiones para el Desarrollo de la Relación. Universidad - Empresa. España.

Galván, J., \& García, P. (2007).La Administración Electrónica en España. Volumen 5 de colección fundación telefónica. Edición ilustrada. Editor INAP. España.

Hidalgo, A., León, G., \& Pavón, J. (2004).La Gestión de la Innovación y la Tecnología en las Organizaciones. Economía y Empresa Series. Edición Ilustrada. Pirámide Editor.

Husted, J., Cook, R., Farewell, V., \& Gladman, D. (2000) Methods for assessing responsiveness: a critical review and recommendations. J Clin Epidemiol, 459-68.

Monteagudo, R. (2005).Didáctica del Planeamiento Estratégico. Ediciones Díaz de Santos.

Morrisey, G. L. (1996). Pensamiento Estratégico: Construya los cimientos de su planeación. Prentice - Hall Hispanoamericana, S.A. México.

Pozo, J. (2004). Teorías Cognitivas del Aprendizaje. Madrid: Morata.

Rodríguez, R. (2004).Cultura de la Innovación y la Gestión Tecnológica para el Desarrollo de los Pueblos. Editor Convenio Andrés Bello.

Senge, P. (1990). La Quinta Disciplina. Cómo Impulsar el Aprendizaje en la Organización Inteligente, Ediciones Juan Granica, España. 